\title{
Accurate Representations of Digital Copyslides: The colour of Yves Klein
}

\author{
Jon Malis \\ Loyola University Maryland \\ 4501 N Charles St, Baltimore, MD \\ 21211 USA \\ idmalis@loyola.edu
}

\section{INTRODUCTION}

Artists have long obsessed over the colours they employ in their work, often going to great lengths to create unique combinations of pigments to achieve specific effects. From 1957 through his death in 1962, the French artist Yves Klein's works were dominated by a specific shade of ultramarine blue International Klein Blue. Developed throughout the 1950's in collaboration with Edouard Adam, an art material and hardware supplier, its specific composition of ultramarine pigment and synthetic binder was patented in 1960 through the use of an enveloppe soleau (a French "proof of invention") (Mancusi-Ungaro 1982; Stitch 1994).

IKB differs from traditional oil and acrylic paints in that the binder it uses to suspend its pigment molecules - Rhodopas M - does not significantly alter the brightness, brilliance and vibrance of the pigment when compared to its pure form. (MancusiUngaro 1982). It is this same combination of pigment and binder that when combined, creates a depth to the work that cannot be truly reproduced digitally - one must view the work in person to truly experience the power of IKB (Ottmann 2007).

With this impossibility of experiencing true IKB in mind, this paper examines the colour accuracy of digitally available reproductions of Klein paintings that exist on publicly accessible and searchable websites.

\section{INTERNATIONAL KLEIN BLUE AS A STANDARD}

As the formulation and composition of IKB is known, the IKB recipe survives as an exacting standard to which his works and their reproductions can be compared. In itself, IKB was not a colour, but rather, a process for which Synthetic Ultramarine Blue pigment number 1311 was dispersed within a medium to create a desired visual effect (Stitch 1994). The veracity of the resulting works' colourmetric data rests within the Synthetic Ultramarine Pigment 1311, as that was Klein's target blue. Ultramarine 1311 is no longer produced, however the shop owned by Adam's family currently supplies a close contemporary version, which is sold separately, and in kits with Medium Adam25 (their version of Rhodopas M) as a "Blue Kit" (Adam E-Shop).

However, use of the Adam "Blue Kit" would not take into account any properties of aging present within Klein's original works, and thus, a better method of determining a reference for IKB would be to analyse and measure the colourmetric data of original Klein paintings. While I have not yet had the opportunity to do so, this would provide the best dataset for equating digitised versions of Klein's paintings against their physical counterparts (see Future Research). In addition to compensate for any aging properties, direct measurement would allow for a range of typical "gallery viewing" conditions to be accounted for (D50 vs Tungsten lighting, curatorial and conservational decisions regarding illumination levels, the presence of windows within the gallery to name a few variables).

Without access to either Adam's Blue Kit nor Klein originals, this paper then looks to other sources to define the digital equivalent of IKB. One researcher posited an RGB equivalent of IKB to being [0, 47, 167], assumed in an SRGB colour space (Grais). RGB.to defines "International Klein Blue" as [69, 68, 147] (RGB.to). With these conflicting definitions, and the inherent profile-based relativity of the RGB colourspace, for the context of this paper, I have chosen a fourth option for generating comparison data - using the images themselves. As I have found images from multiple types of sources (museums, galleries, auction houses, fan sites), I chose to use the images published digitally 
by the Yves Klein Archives (http://www.yvesklein.com/) as references against which all other sources could be measured. While this approach might be lacking in ultimate precision, it provides the most uniform base for all other sources to be measured.

\subsection{The effect of colourspaces in rendering IKB}

As the "default [RGB] colourspace of the Internet", sRGB defines a subset of all visible colours that are most likely able to be viewed accurately on the majority of digital display devices in use globally (Stokes et al. 1996). Owing to this universality, the profile specifies a relatively small gamut (range of colours) that can be accurately portrayed, many highly saturated colours are clipped, and a wide portion of the visible deep blues, greens, and reds are rendered out of gamut (unreproducible).

Using the averaged colourmetric data of the Klein Archives' virtual holdings, a CIELAB value of [L*25.0, $\left.a^{*}-15.9, b^{*}-65.0\right]$ is achieved, which is out of gamut for SRGB, as well as many other RGB colourspaces. Using basic gamut warnings in Adobe Photoshop to indicate in/out of gamut mapping, IKB cannot be viewed in SRGB, Adobe RGB (1998), ColorMatch RGB, and P3. It is ingamut in ProPhoto RGB, as well as e-sRGB and ACES.

All the images I found on the Internet were profiled for either SRGB or Adobe RGB, or were untagged and assumed to be SRGB. As many consumer displays cannot display beyond Adobe RGB, it is a safe assumption that, given the working virtual definition of IKB in this paper, it is impossible to be rendered accurately in the majority of noncalibrated/colour managed settings.

\section{MEASURING THE COLOURMETRIC DATA OF ONLINE AND ACCESSIBLE KLEIN IKB PAINTINGS}

In this study, I have identified 30 unique digital images of Klein IKB paintings (I chose not to include his sponge and irregular works due to their dimensionality and potential for shadows to affect the measurements) of 29 paintings from 7 sources: Yves Klein's Archive, the Tate, Museu Coleção Berardo, MoMA, Christie's, Artsy and Artnet (one painting, IKB 234 appears in the dataset twice, as Christies documented the work for a 2006 auction, and then again when it re-appeared at auction in 2008). The majority of these images are embedded with either the Adobe RGB (1998) colour profile or are untagged, and thus are assumed to be viewed in SRGB and are assigned as such at open. To obtain representative $L^{*} a^{*} b^{*}$ and $R G B$ values for each work, the images were blurred with a 25-pixel Gaussian Blur to average any variances in texture and subtle shadow (as many slides contained white borders and edges, an overall average blur function produced skewed averages which included the borders), and then representative values were samples from three spots in the middle of the image. These three values were averaged and recorded to obtain the data used in this analysis.

Eleven works in the dataset came from the Klein Archive's website, and thus, this grouping of 11 was used to generate the "average" $L^{*} a * b *$ values for Klein works referenced above. Once these averages were computed, a statistical analysis was applied to the data, computing the $\Delta \mathrm{E}$ (DeltaEmpfindung, or "difference in sensation") between the dominant colour in each image and the averaged reference. A number of different DeltaE formulas exist, this study employed DeltaE 2000 (Lindbloom 2017) unless otherwise noted. When comparing two works, the $\Delta \mathrm{E}$ scale is as follows: $<1$ : not perceptible to human eyes, 1-2: perceptible via close observation, 2-10: difference perceptible at a glance, 11-49: colours are more similar than different, 100: colours are exact opposites. (Schuessler 2019). When averaged together, the $\Delta$ E2000 for the Klein Archives' collection was 3.13, with three works measuring under one and only one work measuring over 10.

Table 1: Images Sourced from Yves Klein Archive

\begin{tabular}{|l|l|}
\hline \multicolumn{1}{|c|}{ Title } & \multicolumn{1}{c|}{$\Delta \mathbf{E}$} \\
\hline IKB 129 & 15.78 \\
\hline IKB 103 & 0.61 \\
\hline IKB 216 & 2.03 \\
\hline IKB 130 & 3.74 \\
\hline IKB 104 & 0.85 \\
\hline IKB 100 & 0.95 \\
\hline IKB 75 & 2.75 \\
\hline IKB 167 & 1.04 \\
\hline IKB 47 & 2.96 \\
\hline IKB 38 & 1.68 \\
\hline
\end{tabular}

The largest dataset came from Christie's auction house, with 14 images of 13 works. The average $\Delta \mathrm{E}$, when compared to the Klein archives, was 23.22, with the best 5.99 (IKB 93) and the worst 57.39 (IKB 319); the majority computed within 4 points of the average. A unique element in this dataset is that the same painting (IKB 234) appears twice, as it was documented on two separate occasions in 2006 and 208 for separate sales; the $\Delta \mathrm{E}$ for these two images of the same work calculates to 5.95 . 
Table 2: Images Sourced from Christie's

\begin{tabular}{|l|l|}
\hline \multicolumn{1}{|c|}{ Title } & $\Delta \mathrm{E}$ \\
\hline IKB 319 & 57.37 \\
\hline IKB 311 & 22.91 \\
\hline IKB 276 & 18.95 \\
\hline IKB 240 & 23.33 \\
\hline IKB 234 (2006 sale) & 23.27 \\
\hline IKB 234 (2008 sale) & 17.54 \\
\hline IKB 217 & 17.08 \\
\hline IKB 182 & 23.30 \\
\hline IKB 146 & 21.30 \\
\hline IKB 163 & 23.17 \\
\hline IKB 108 & 17.68 \\
\hline IKB 107 & 26.41 \\
\hline IKB 93 & 6.00 \\
\hline IKB 77 & 57.37 \\
\hline
\end{tabular}

\section{IS "AT A GLACE" GOOD ENOUGH?}

As discussed above, it is impossible to correctly render IKB within the SRGB colourspace, which is the fundamental baseline for any sort of device consistency. Although it can be rendered in ProPhoto, most modern hardware cannot support any gamuts wider than P3, Adobe RGB and Rec2020, which are all still smaller than ProPhoto. Using the $\Delta \mathrm{E}$ data from above, we can compare the images to the standard of "Just Noticeable Different", which is $2.3 \Delta \mathrm{E}$ units. (Mokrzycki \& Tatol 2011) Using that as a guide, 7 of the 9 images from the Yves Klein archive fall within the window of the colloquial norm, while only one image from other sources scores under 5 .

Assuming that the majority of viewing environments are uncontrolled (no colour calibration, device profiling or software rendering) using a $\Delta \mathrm{E}$ of under 10 (the upper limits of "difference perceptible at a glance") 13 of the 30 images are represented.

At this stage and without surveying a number of original Klein works, it is impossible to determine what an acceptable $\Delta \mathrm{E}$ value is for viewing his work, especially as it is not known how accurate the initial data found in the Yves Klein Archive's images is.

\section{NEXT STEPS AND FUTURE RESEARCH}

As discussed throughout, this project is very much a work in progress. While the initial study's goals of comparing digital representations of Klein's work from a variety of sources to test for colour accuracy were met, it was quickly realised that competing colour approximations exist on the internet for Klein's work, and there is no published research containing photo/colourmetric data of his works insitu.

The next logical step, then, is to identify institutions and collections holding Klein paintings that would allow for non-contact readings of their work's colourmetric properties, ideally under controlled and/or typical gallery lighting conditions. If a large enough dataset is able to be constructed, this information could potentially be beneficial to understanding the colour-based effects of aging in Klein's blue works, documenting colour shifts and correlating to storage/handling/presentation conditions. Given the relative short timespan in which he was producing these works coupled with Adam's documented formula, cataloguing the variations that exist might prove of interest to the greater study of Klein's works and legacy.

Once that colourmetric information is derived, further study and analysis of Klein's digital copyslides can begin, with a better, known (not estimated) $L^{*} a^{*} b^{*}$ reference to compare all images to.

\section{REFERENCES}

Adam E-Shop (2020) Blue Kit. http://www.adameshop.com/en/539-blue-kit (retrieved 5 March 2020).

Christie's | Yves Klein. https://artist.christies.com/YvesKlein-30158.aspx (retrieved 5 March 2020).

Grais, S. (2020) International Klein Blue. http://facweb.cs.depaul.edu/sgrais/international klein blu e.htm (retrieved 5 March 2020).

Lindbloom, B. (2017) Delta E (CIE2000). http://www.brucelindbloom.com/Eqn DeltaE CIE2000.ht $\underline{\mathrm{ml}}$ (retrieved 5 March 2020)

Mancusi-Ungaro, C. (1982) A Technical Note on IKB. In Yves Klein 1928-1962: A Retrospective, Institute for the Arts, Rice University, Houston, TX, pp.258-9

Mokrzycki W. S., and Tatol M. (2011) Colour difference $\Delta \mathrm{E}-\mathrm{A}$ survey. Machine Graphics \& Vision International Journal. 20(4), pp.383-411.

Ottmann, K. (2007) Experience or Interpretation: "What You See Is Not What You Read". The Journal of Aesthetic Education. 41(2), pp.13-17

RGB.to. (2020) Convert Colour International Klein Blue. https://rgb.to/colour/9652/international-klein-blue (retrieved 5 March 2020).

Schuessler, Z. (2019) Delta E 101. http://zschuessler.github.io/DeltaE/learn/ (retrieved 5 March 2020).

Stitch, S. (1994) Yves Klein. Hatje Cantz, Stuttgart, Germany.

Stokes, M., Anderson, M., Chandrasekar, S., and Motta, R. (1996) A Standard Default Colour Space for the Internet sRGB. https://www.w3.org/Graphics/Colour/sRGB.html (retrieved 5 March 2020).

Yves Klein Archive http://www.yvesklein.com (retrieved 5 March 2020). 